\title{
Optical properties of gold and aluminium nanoparticles for silicon solar cell applications
}

\author{
T. L. Temple a) and D. M. Bagnall \\ Electronics and Computer Science, University of Southampton, Highfield, Southampton, SO17 1BJ, \\ United Kingdom
}

(Received 17 December 2010; accepted 10 March 2011; published online 27 April 2011)

\begin{abstract}
The optical properties of metal nanoparticles are explored as a function of lateral size, shape, aspect-ratio and metal type. Simulations based on the discrete dipole approximation are compared with experimental measurements of arrays of metal nanoparticles fabricated by electron-beam lithography. Careful selection of experimental parameters ensures minimization of far-field and near-field coupling, and inhomogeneous broadening, thus allowing comparison with single particle simulations. The optical properties of Au nanoparticles are compared with similar Al nanoparticles for each particle type. For solar cell light-trapping applications, we require metal nanoparticles that exhibit extinction peaks near the band-edge region of the absorbing material, as well as low absorption and large optical cross-sections. Al nanoparticles are shown to be of interest for amorphous silicon solar cells, but their applications for polycrystalline solar cells is limited by the presence of an interband region in the near-infrared. The opposite is found for Au nanoparticles, which feature an interband threshold region in the visible that makes their optical properties unsuitable for amorphous silicon but very suitable for crystalline and polycrystalline silicon solar cells. @ 2011 American Institute of Physics. [doi:10.1063/1.3574657]
\end{abstract}

\section{INTRODUCTION}

Metal nanoparticles interact strongly with visible and infrared photons due to the excitation of localized surface plasmons (LSPs). ${ }^{1,2}$ LSPs are a result of coherent oscillations of conduction electrons, and can be excited in some metals by UV, visible or NIR photons. The strongest optical interaction occurs at a resonance, with the resonance condition being a function of the nanoparticle size, shape, and type of metal, as well as the local dielectric environment. ${ }^{3}$ Once excited an LSP can decay radiatively, resulting in scattering, or nonradiatively, resulting in absorption. The sum of absorption and scattering is known as extinction, and the extinction peak occurs at the resonant wavelength of the LSP. The strong, tuneable optical properties of metal nanoparticles have lead to a wide range of applications, ranging from biosensing ${ }^{4,5}$ to photovoltaics. ${ }^{6,7}$

In the case of silicon photovoltaics, metal nanoparticles offer the prospect of increasing device efficiency by reducing surface reflectance ${ }^{8}$ and/or increasing light-trapping within thin-film devices. ${ }^{9,10}$ However, metal nanoparticles can also decrease the efficiency of solar cells, for example due to absorption of light within the nanoparticle ${ }^{11}$ or by increasing reflectance of the front surface due to back-scattering. ${ }^{12,13}$ Therefore, it is imperative that metal nanoparticles are suitably designed to provide the correct optical properties for a given application.

In order to enhance light-trapping in silicon solar cells we require nanoparticles that exhibit low absorption in the visible and near-infrared (NIR), and large scattering crosssections across the useful solar spectrum. It is particularly

${ }^{\text {a)} E l e c t r o n i c ~ m a i l: ~ t l t @ e c s . s o t o n . a c . u k . ~}$ important to achieve high scattering near the band-edge regions of thin film amorphous silicon $(\sim 715 \mathrm{~nm})$ or thin film polycrystalline silicon $(\sim 1100 \mathrm{~nm})$, as this is where these materials have a low absorption coefficients and solar cell quantum efficiencies are low. Importantly, light-trapping in the NIR is difficult to achieve with conventional surface texturing approaches, since the dimensions of the texturing must be comparable to the wavelength of interest in order to scatter efficiently. However, high surface roughness can lead to poor quality film growth or the formation of shunting paths, resulting in a reduction of the efficiency of thin film photovoltaic devices. ${ }^{14,15}$ By contrast, metal nanoparticles can strongly scatter light despite having dimensions substantially smaller than the incident wavelength. Therefore, metal nanoparticles could remove the need for rough textured surfaces while providing improved light-trapping; however, if utilized incorrectly they could introduce additional loss mechanisms. These additional loss mechanisms must be minimized by the development of a complete understanding of the optical properties of particles of different size, shape and metal type.

\section{BACKGROUND}

The extinction efficiency is the ratio of the optical crosssection to the geometric cross-section of the nanoparticle. Metal nanoparticles typically have peak extinction efficiencies that greatly exceed unity, such that even an incomplete surface coverage of particles can scatter and/or absorb all incident photons. For example, a nanoparticle with an extinction efficiency of 5 requires only a $20 \%$ surface coverage for complete interaction with incident photons. Larger extinction efficiencies mean that fewer nanoparticles (and hence a lower surface coverage) are required. 
The ratio of scattering to extinction is known as the radiative efficiency, and ranges from zero (completely absorbing) to one (completely scattering). In most circumstances any light absorbed by the nanoparticles will not generate photocurrent, and so the radiative efficiency must be as close to unity as possible.

Studies involving metal nanoparticles and silicon solar cells have tended to focus on $\mathrm{Au}$ or $\mathrm{Ag}$ nanoparticles with spherical ${ }^{8}$ or "island" (sometimes approximated as truncated spheroid) geometries. ${ }^{9-11}$ These metals and nanoparticle geometries represent only a small sub-section of the available parameter space, and further optimization of the nanoparticle size, shape, and metal is likely to result in significant enhancement of photovoltaic devices.

Localized surface plasmons can only be efficiently excited at energies where the metal does not have significant interband transitions available, i.e., at energies where freeelectron (Drude-like) behavior is dominant. Interband transitions offer additional decay paths for excited electrons, and hence either prohibit or damp the excitation of LSPs. The noble metals $(\mathrm{Ag}, \mathrm{Au}$, and $\mathrm{Cu}$ ) support excitation of LSPs when excited by photons with energy lower than the interband transition thresholds of approximately $3.8 \mathrm{eV}, 2.4 \mathrm{eV}$ and $2.1 \mathrm{eV}$ for $\mathrm{Ag}, \mathrm{Au}$, and $\mathrm{Cu}$, respectively. ${ }^{16}$ Conversely, $\mathrm{Al}$ supports excitation of LSPs above and below a narrow interband transition range centered around $1.5 \mathrm{eV} .{ }^{17}$ The alkali metals also support LSPs in the visible and NIR ${ }^{18}$ but are not suitable for PV applications due to their low chemical stability. Other metals such as Pt and Pd are unsuitable for silicon solar cells due to the presence of interband transitions across the visible and NIR and correspondingly poor radiative efficiencies in this range. ${ }^{19}$

Al nanoparticles feature markedly different optical properties to the noble metals ${ }^{20}$ and have been shown to support resonances at much shorter wavelengths, suggesting promise for UV applications such as SERS (Refs. 21, 22) and metalenhanced fluorescence. ${ }^{23} \mathrm{Al}$ is usually not considered for photovoltaic applications, often because it is assumed that $\mathrm{Al}$ nanoparticles only support resonances in the UV (Ref. 24); this is despite the fact that experiments using disclike ${ }^{25}$ and triangular $^{20}$ geometries have shown that Al nanoparticles are capable of supporting LSPs ranging from the UV to the NIR. In both cases the peak position is changed by increasing the lateral particle shape; however, the influence of size and shape on $\mathrm{Al}$ nanoparticles and their potential for applications at longer wavelengths (such as in photovoltaics) have not yet been fully investigated.

Achieving high scattering efficiencies in the NIR with spherical nanoparticles requires large particles (diameter $>150 \mathrm{~nm})^{26}$ and/or a surrounding medium with a high refractive index. Large particles increase the surface roughness of the substrate, which is not desirable for thin-film solar cells. An alternative is to change the particle shape rather than the size in order to red-shift the scattering peak to the desired wavelength range. This enables tuning of the peak position far into the NIR without introducing substantial surface roughness.

In this work we investigate the optical properties of $\mathrm{Au}$ and $\mathrm{Al}$ nanoparticles as a function of size and shape. The work is carried out with particular concern for photovoltaic applications, where we are interested in maximizing the extinction efficiency and radiative efficiency in the visible and NIR regions of the solar spectrum, and particularly near the band-edges of amorphous silicon and polycrystalline silicon. Simulations based on the discrete dipole approximation (DDA) are compared with experimental measurements of arrays of metal nanoparticles fabricated by electron-beam lithography (EBL). Careful selection of experimental parameters ensures minimization of far-field and near-field coupling, and inhomogeneous broadening, thus allowing comparison with single particle simulations.

\section{EXPERIMENTAL DETAILS}

\section{A. Fabrication}

Electron-beam lithography (EBL) was used to fabricate random arrays of metal nanoparticles. EBL minimizes inhomogeneous broadening, and allows nearly identical particles to be fabricated using a variety of metals. However, EBL is a serial process-each particle must be defined individuallyand so is unsuitable for fabricating the large-area arrays required for photovoltaic applications. We make use of EBL to obtain experimental clarity and flexibility, but we restrict our consideration to simple geometries that we believe could be fabricated by low-cost, large-area methods in the near future.

The $1.5 \times 1.5 \mathrm{~mm}^{2}$ arrays of metal nanoparticles were fabricated using conventional EBL and lift-off. Patterns were defined in ZEP520 resist layers, working with a spot size of $4 \mathrm{~nm}$ and an accelerating voltage of $100 \mathrm{kV}$. ZEP520 was spun-cast onto $150 \mathrm{~mm}$ diameter fused-silica wafers with a layer thickness of around $115 \mathrm{~nm}$. A $20 \mathrm{~nm}$ Al layer was then deposited to avoid charging of the substrate during exposure. Each array contains 9,006,001 particles, corresponding to an average center-to-center separation of $500 \mathrm{~nm}$. Particle dimensions range from $50 \mathrm{~nm}$ to $200 \mathrm{~nm}$, and the metal layer thickness (i.e., out-of-plane particle height) is 40 $\mathrm{nm}$ for all samples.

With the exception of the results presented in Sec. III A, all results in this work are for random arrays of nanoparticles. The random positioning of nanoparticles was achieved by displacing coordinates of each nanoparticle in the mask layout by randomly chosen $\mathrm{x}$ and $\mathrm{y}$ offsets ranging from $500 \mathrm{~nm}$ and $500 \mathrm{~nm}$. An SEM image of a typical array is given in Fig. 1.

To reduce near-field coupling, the minimum allowed center-to-center spacing was restricted to $350 \mathrm{~nm}$ for the 200 $\mathrm{nm}$ square particles, and $300 \mathrm{~nm}$ for all other particles. The number of particles in each array was kept constant, and so the average interparticle separation will decrease as the nanoparticle size is increased. Previous studies have shown that near-field coupling affects the optical properties of nanoparticles for edge-to-edge separations of the order of the radius of the nanoparticle. ${ }^{27}$ Table I lists the nanoparticle dimensions, surface coverage and interparticle separation of the fabricated arrays used in this study. With the exception of the largest square nanoparticle, all of the arrays have average minimum interparticle separations exceeding the length 


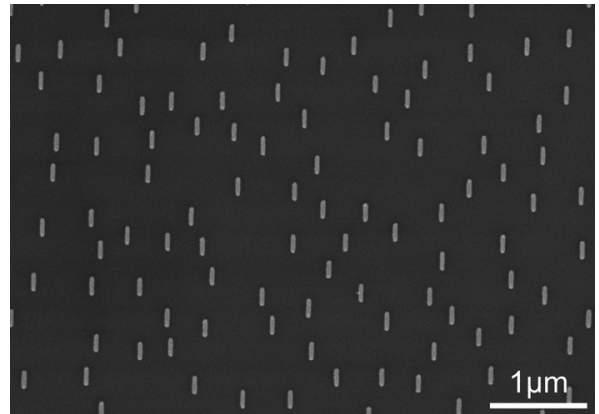

FIG. 1. SEM image showing the arrangement of a typical array of nanoparticles.

of the nanoparticle, and fewer than $1.75 \%$ of the nanoparticles have a nearest neighbor closer than $100 \mathrm{~nm}$. As such we expect there to be minimal near-field coupling in these arrays. The $200 \mathrm{~nm}$ square nanoparticles have an average minimum interparticle separation less than the side length, and $6.54 \%$ are less than $100 \mathrm{~nm}$ from their nearest neighbor (edge-to-edge). As such there may be a modest shift in peak position for some nanoparticles in this array, but we expect the majority of the signal to be from nanoparticles in the array that are not affected by near-field coupling. Further investigations are required to ascertain the maximum surface coverage that can be achieved for each nanoparticle shape before near-field coupling alters the response of an array away from that of an isolated nanoparticle.

Identical designs were used for both $\mathrm{Au}$ and $\mathrm{Al}$ nanoparticles. Au does not adhere well to silica and so an adhesion layer is required to prevent complete removal of metal during lift-off. $\mathrm{Ti}$ and $\mathrm{Cr}$ layers are often used to improve adhesion of noble metals to surfaces, but these layers also degrade the optical properties of metal nanoparticles and so their thickness must be minimized. ${ }^{28}$ As such, a $\mathrm{Cr}$ layer with nominal thickness of $3 \mathrm{~nm}$ was used in this experiment. Arrays of Al nanoparticles were successfully fabricated without an adhesion layer.

\section{B. Characterization}

Optical measurements are made using bespoke fibercoupled transmission apparatus. Light from a tungsten-halogen bulb (Bentham IL1) is coupled to a $100 \mu \mathrm{m}$ core silica

TABLE I. List of details of the fabricated nanoparticle arrays.

\begin{tabular}{lccc}
\hline \hline & $\begin{array}{c}\text { Surface } \\
\text { Coverage } \\
(\%)\end{array}$ & $\begin{array}{c}\text { Minimum } \\
\text { nearest } \\
\text { neighbour } \\
\text { distance }(\mathrm{nm})\end{array}$ & $\begin{array}{c}\text { Average } \\
\text { nearest } \\
\text { neighbour } \\
\text { distance }(\mathrm{nm})\end{array}$ \\
\hline $100 \mathrm{~nm} \times 100 \mathrm{~nm}$ square & 4.00 & 159 & 255 \\
$150 \mathrm{~nm} \times 150 \mathrm{~nm}$ square & 9.00 & 88 & 197 \\
$200 \mathrm{~nm} \times 200 \mathrm{~nm}$ square & 16.00 & 67 & 174 \\
$50 \mathrm{~nm} \times 100 \mathrm{~nm}$ rectangle & 2.00 & 188 & 283 \\
$50 \mathrm{~nm} \times 150 \mathrm{~nm}$ rectangle & 3.00 & 142 & 252 \\
$50 \mathrm{~nm} \times 200 \mathrm{~nm}$ rectangle & 4.00 & 95 & 219 \\
$115 \mathrm{~nm}$ diameter circle & 4.15 & 185 & 261 \\
$150 \mathrm{~nm}$ side length triangle & 3.90 & 150 & 236 \\
\hline \hline
\end{tabular}

fiber (Ocean Optics) and focused onto the sample using a $100 \mathrm{~mm}$ focal length lens to form a spot smaller than the sample size. Light from the input fiber is directed into a 600 $\mu \mathrm{m}$ silica fiber (Ocean Optics) by a collimating lens with a small acceptance angle to minimize detection of scattered light. This fiber is connected directly to a VIS-NIR spectrometer (HR4000, Ocean Optics). Measurements are normalized to the transmission of the uncoated areas of the fused-silica substrates.

Size and shape of the fabricated nanoparticles was performed using SEM inspection. To avoid charging on the silica substrates and associated degradation of image quality, SEM inspection was performed on identical arrays of $\mathrm{Au}$ nanoparticles fabricated on a silicon wafer coated with a 30 $\mathrm{nm} \mathrm{SiO}_{2}$ layer.

Extinction efficiencies are calculated by dividing the extinction percentage by the surface coverage. The surface coverage was calculated from the cross-sectional area of the corresponding nanoparticle design. The fabricated particles generally have a lower cross-sectional size than designed, and as such we expect the experimental extinction efficiencies to be slightly underestimated.

\section{Simulation}

The discrete dipole approximation (DDA) ${ }^{29,30}$ can be used to simulate the optical properties of metal nanoparticles with arbitrary size and shape. In this method the particle is approximated as a 3D lattice of polarisable point dipoles, and Maxwell's equations are then solved directly. DDA yields accurate results provided the interdipole separation is substantially smaller than the incident wavelength, and also small enough such that the dipoles can sufficiently represent the geometrical features of the particle. ${ }^{27}$ An open-source implementation of the DDA, DDSCAT 6.1, is used in this work. ${ }^{31}$

Each model contains between 20,000 and 50,000 dipoles to ensure that the target geometry is suitably represented and that accurate results are obtained. Optical constants for $\mathrm{Al}$ and $\mathrm{Au}$ were obtained from Palik, ${ }^{17}$ and fitted using parabolic interpolation. The simulations are performed with a wavelength step of $10 \mathrm{~nm}$, with light at normal incidence to the particle. The spectra presented are the average between two orthogonal polarizations, which approximate the response of the particle to unpolarized light, as is used in the experiment. DDSCAT 6.1 calculates optical efficiencies by dividing the calculated cross-section by the cross-sectional area of a sphere with the same volume as the target geometry. This can lead to misleadingly large efficiencies, particularly for flat particles with a high surface to volume ratio. As such, "correct" optical efficiencies were obtained by modifying the program to divide the calculated cross-section by the true cross-sectional area of the nanoparticle.

The accuracy of the DDA simulations was tested by comparing the results with Mie theory, ${ }^{32}$ which is an exact solution to Maxwell's equations for the case of a sphere. The same optical constants and interpolation routines were used for each method, and a comparison was made for $\mathrm{Al}$ and $\mathrm{Au}$ spheres with a diameter of $50 \mathrm{~nm}, 100 \mathrm{~nm}$ and $150 \mathrm{~nm}$ 

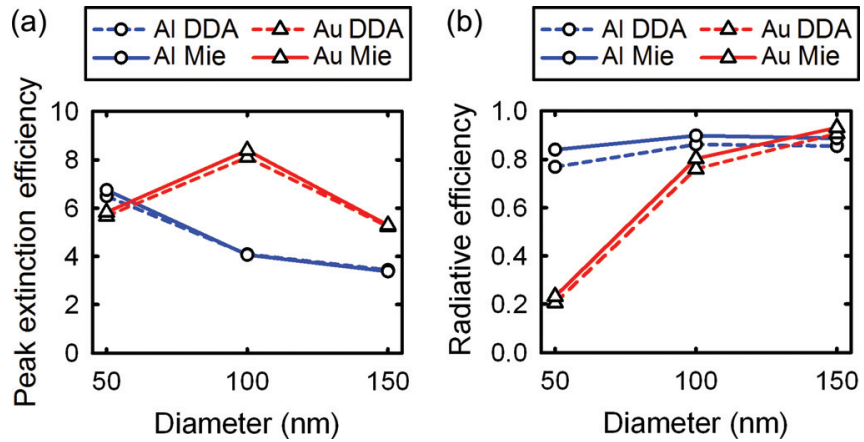

FIG. 2. (Color online) Comparison of DDA and Mie theory calculations for $\mathrm{Al}$ and $\mathrm{Au}$ spheres, with diameters of $50 \mathrm{~nm}, 100 \mathrm{~nm}$, and $150 \mathrm{~nm}$, embedded in a homogenous medium with a refractive index of 1.5 . Trends are presented for (a) peak extinction efficiency and (b) radiative efficiency.

(Fig. 2). The number of dipoles used in the DDA models was $18,853,157,563$ and 492,968 , which correspond to a grid size (i.e., interdipole spacing) of approximately $1.5 \mathrm{~nm}$ for all three models. Excellent agreement was found between the extinction peak positions calculated by DDA and Mie theory, with an error margin smaller than the $10 \mathrm{~nm}$ wavelength step used in the calculation. The extinction efficiencies are also in good agreement, with an error margin of the order of a few percent [Fig. 2(a)]. However, the radiative efficiencies calculated by DDA are between $2.3 \%$ and $10.5 \%$ lower than those calculated by Mie theory [Fig. 2(b)]. The largest errors occur for the smallest particles. The reason for the error is most likely the granularity of the approximation of the particle surface in the DDA models, which leads to erroneous field hot spots. ${ }^{3,27}$ The smaller particles have a larger surface-to-volume ratio and so are more strongly affected by errors arising from surface dipoles. ${ }^{30,33}$ Therefore, we conclude that the peak position and extinction efficiency calculated by DDA can be considered as accurate, but the calculated radiative efficiency can be more than $10 \%$ lower than the true value and must be treated with some caution.

\section{RESULTS AND DISCUSSION}

\section{A. Periodicity}

Nanoparticles fabricated using EBL are typically arranged in a periodic grid. However, this introduces diffractive orders, and so the array can exhibit markedly different optical properties from those of each individual nanoparticle. $^{34}$ To demonstrate the influence of array structrue, arrays of square nanoparticles with a side length of $150 \mathrm{~nm}$ were fabricated with either a square or a random structure. The square array has a pitch of $500 \mathrm{~nm}$, and the random array has the same surface coveraged but a disordered array structure that was achieved by moving each particle from its position in the array by a random $\mathrm{x}$ and $\mathrm{y}$ offset.

Figure 3 shows that, as expected, the optical properties of the periodic and random arrays are markedly different. The random arrays feature broad, roughly symmetric peaks, while the ordered arrays feature narrower, assymmetric peaks. The changes in the extinction spectra are predominantly due to the suppression and enhancement of scattering at different wavelengths due to diffractive orders in the periodic array. ${ }^{35}$

The influence of array structure on the optical properties of metal nanoparticles is well studied and can lead to interesting optical properties such as ultra-narrow extinction peaks. ${ }^{36,37}$ However, to simplify our investigation and maintain relevance to bottom-up fabrication techniques, we will focus on random particle arrangement for the remainder of this article. In this case comparison of experimental results with single particle simulations is valid, provided the minimum interparticle spacing of the fabricated arrays is such that near-field coupling is minimized.

\section{B. Surrounding medium}

The DDA can be used to model the optical properties of metal nanoparticles embedded in a nonabsorbing, homogenous medium. However, the dielectric environment in practical experiments is more complicated. In this section we will use the example of three nanorods, with width $50 \mathrm{~nm}$ and lengths of $100 \mathrm{~nm}, 150 \mathrm{~nm}$, and $200 \mathrm{~nm}$ to investigate the influence of the surrounding medium. These dimensions result in extinction peaks spanning across a wide wavelength range. The optical properties of nanorods themselves will be discussed in more detail in Sec. IV E.

The largest difference between the dielectric environments of the simulations and experimental particles is the presence of a fused-silica substrate. Fused-silica is transparent across the wavelength range used in the experiments, but it has a higher refractive index than vacuum and so will have an influence on the optical properties of the metal nanoparticles. Kelly et al. included the effect of silicon and carbon substrates in DDA models by means of a hemisphere encompassing the near-field region of the substrate side of the nanoparticle ${ }^{3}$ however, this introduces considerable computational complexity, and the overall optical properties will be partially determined by the size of the substrate hemisphere.

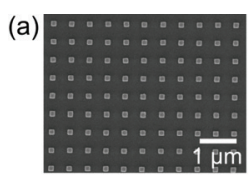

(b)

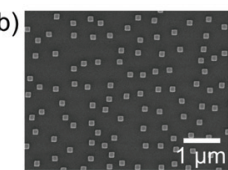

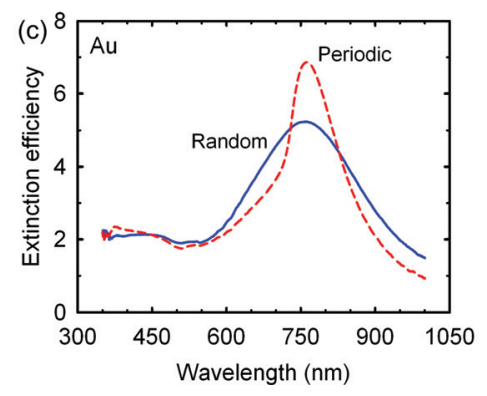

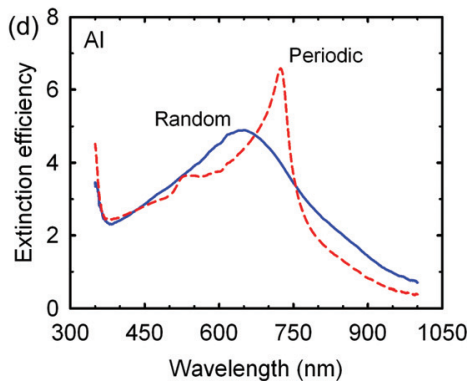

FIG. 3. (Color online) SEM images of (a) periodic arrays and (b) random arrays of square particles with a side length of $150 \mathrm{~nm}$. (c) Extinction spectra of $\mathrm{Au}$ arrays, (d) extinction spectra of $\mathrm{Al}$ arrays. 
(a)

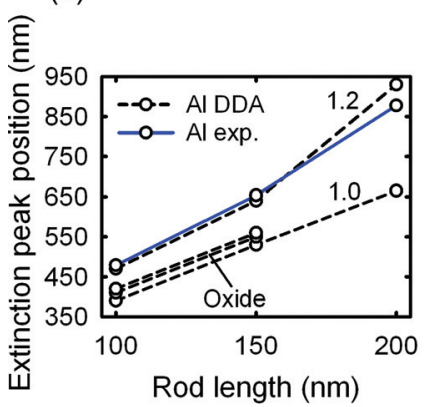

(b)

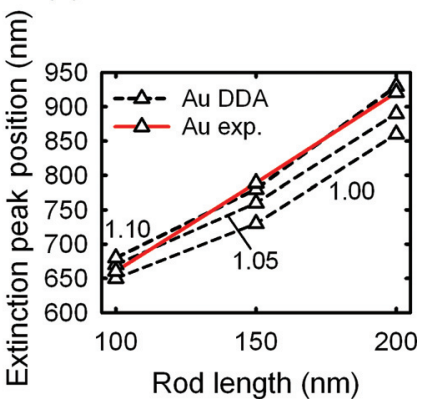

FIG. 4. (Color online) Fitting of DDA simulations to experimental results. (a) Al nanorods in a homogeous media of refractive index 1.0 and 1.2, and the influence of replacing the outer $2 \mathrm{~nm}$ and $3 \mathrm{~nm}$ of the particle surface with an oxide layer. (b) Au nanorods in a media of refractive index 1.00 , 1.05 , and 1.10 . The rod width is $50 \mathrm{~nm}$ in all cases.

Instead, we choose to approximate the substrate by a homogenous surrounding medium with a refractive index between that of the substrate and air. The refractive index of this medium was determined using a simple iterative fit to the experimental data, as shown in Fig. 4. Good agreement with experimental data was found for a surrounding medium with refractive index of 1.2 for $\mathrm{Al}$ nanoparticles and 1.1 for $\mathrm{Au}$ nanoparticles. These values lie between that of air (1.0) and the substrate (1.5), as expected. The values are closer to that of air because more of the particle surface is in contact with air than with the substrate. We note that the obtained refractive index values may also partially derive from other discrepancies between the simulations and experiments aside from the dielectric environment. For example the fabricated nanoparticles are slightly smaller than those in the experimental model, which will require a lower refractive index homogenous medium in the simulation to blue-shift the peak position to match the experimental spectra.

The difference in refractive index of the fitted effective medium values for $\mathrm{Al}$ and $\mathrm{Au}$ is due to the fact that the local dielectric environments for the two metals are not the same. Al nanoparticles are expected to be coated with a 2-3 nm layer of $\mathrm{Al}_{2} \mathrm{O}_{3}$, which forms spontaneously on contact with air. Langhammer et al. suggest that the oxide growth has two effects on the extinction peak position: a red-shift due to an increase in the local refractive index, and blueshift due to a decrease in the size of the core Al particle. ${ }^{25}$ Figure 4(a) shows the result of replacing the outer surface of the particle surface with either $2 \mathrm{~nm}$ or $3 \mathrm{~nm}$ of $\mathrm{Al}_{2} \mathrm{O}_{3}$, and clearly demonstrates that the red-shift due to the oxide layer dominates the blue-shift due to decreasing the nanoparticle size, resulting in a net red-shift. However, the overall effect of the oxide layer on the peak position is modest compared with the effect of the substrate.

No oxide layer is present on the Au nanoparticles, but in our experiments a $\sim 3 \mathrm{~nm}$ Cr layer was used to improve adhesion to the substrate. Zheng et al. used DDA simulations to show that thin $\mathrm{Cr}$ adhesions layers blue-shift, broaden and attenuate the extinction peak of $\mathrm{Au}$ nanoparticles. ${ }^{28}$ The broadening and attenuation of the peak is due to the $\mathrm{Cr}$ layer damping the LSP, but the reason for the slight blue-shifting is not clear. Figure 5 shows that the simulated extinction effi- (a)

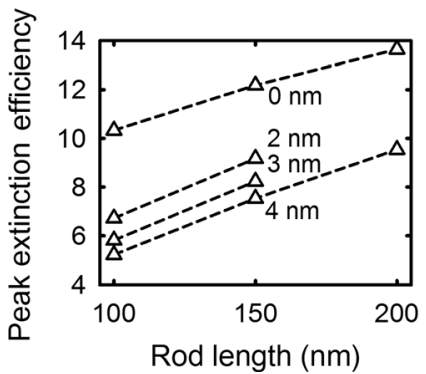

(b)

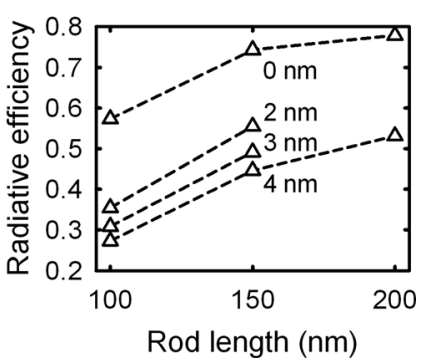

FIG. 5. Simulated spectra showing the influence of $\mathrm{Cr}$ adhesion layers on (a) the peak extinction efficiency and (b) the radiative efficiency of Au nanorods. The Cr layer are situated on the underside of the particle, and have the same cross-section as the Au nanorods.

ciencies and radiative efficiencies of $\mathrm{Au}$ nanorods are both substantially decreased as the Cr layer thickness is increased. For example, the addition of a $4 \mathrm{~nm}$ Cr layer to a $150 \mathrm{~nm}$ by $50 \mathrm{~nm}$ by $40 \mathrm{~nm} \mathrm{Au}$ nanorod reduces the extinction efficiency by $38.5 \%$ and the radiative efficiency by $40 \%$.

The difference between the effective medium values for $\mathrm{Al}$ and $\mathrm{Au}$ is therefore due to the red-shifting and blue-shifting contributions of the $\mathrm{Al}_{2} \mathrm{O}_{3}$ and the $\mathrm{Cr}$ adhesion layer, respectively. Inclusion of thin layers in the simulation model requires a greatly increased number of dipoles, due to the need for a reduced interdipole spacing. This considerably increases the computational requirements, and as such it was not possible to include these layers in the simulation models for all particle geometries, due to memory restrictions in the simulation package used. Instead, for the remainder of this article (with the exception of Sec. IV F) we will make use of simulations of isolated particles embedded in a homogenous medium with a refractive index of 1.1 for $\mathrm{Au}$ and 1.2 for $\mathrm{Al}$. The shift in peak position is well represented by a homogenous medium for both materials, as shown in Fig. 4, but the additional attenuation and damping effects of the ultrathin $\mathrm{Cr}$ layers are not accounted for. Clearly $\mathrm{Cr}$ adhesion layers should be avoided for photovoltaic applications, due to the greatly decreased radiative efficiency. As such our simulations represent what might be achieved if $\mathrm{Au}$ nanoparticles can be fabricated without an adhesion layer, which is challenging with EBL, but possible with other fabrication techniques.

In the following sections we use our simulations and experimental results to explore the most general trends for extinction peak position, peak extinction efficiency and radiative efficiency that we observe when changing the size, shape, and aspect-ratio of $40 \mathrm{~nm}$ thick $\mathrm{Au}$ and $\mathrm{Al}$ planar nanoparticles. In all of these discussions it is important to consider the limitations in the simulation method and the experimental circumstances, most pertinently the $10 \%$ inaccuracy in the simulated value of radiative efficiency and the considerable damping of the experimental Au extinction due to the presence of the $\mathrm{Cr}$ adhesion layer. In spite of these difficulties we have found that these experiments and simulations provide a valuable insight into the main features of metal nanoparticles and as such provide us with an opportunity to consider the specific relevance to photovoltaics. 


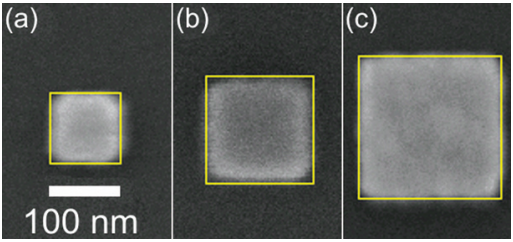

FIG. 6. (Color online) SEM images of typical square nanoparticles fabricated by EBL, with side length of (a) $100 \mathrm{~nm}$, (b) $150 \mathrm{~nm}$, and (c) $200 \mathrm{~nm}$. The yellow outlines show the designed dimensions, drawn to the same scale as the SEM image.

\section{Cross-sectional size}

Increasing the volume of a spherical metal nanoparticle red-shifts and broadens the extinction peak. ${ }^{26}$ The optical properties of lithographically defined nanoparticles can be tuned in a similar manner, for example by increasing the cross-sectional area. The influence of size was investigated by considering square nanoparticles with four side lengths: $50 \mathrm{~nm}, 100 \mathrm{~nm}, 150 \mathrm{~nm}$, and $200 \mathrm{~nm}$. The $50 \mathrm{~nm}$ particles were not fabricated and are only included in the simulation study. SEM images showing examples of typical particles for each array are provided in Fig. 6. The fabricated particles are generally a good match to the designed size and shape, except for some curvature at the corners. The experimental and simulated optical properties of these nanoparticles are shown in Fig. 7.

It can be seen that $\mathrm{Au}$ and $\mathrm{Al}$ nanoparticles both support localized surface plasmons in the visible and NIR, giving rise to distinct extinction peaks. The extinction peak position is broadened and red-shifted with increasing lateral size, as is the case for spheres. Unlike the case for spheres, this tuning is achieved without a change in out-of-plane height, and so the peak-to-peak surface roughness is not increased. This is of importance if the nanoparticles form the substrate upon which a thin-film solar cell is deposited, as rough substrates can lead to poor quality semiconductor film growth.

The peak positions of the experimental spectra of the $\mathrm{Au}$ nanoparticle arrays are in good agreement with the simulation results. However, the experimental extinction peaks for $\mathrm{Au}$ nanoparticles are considerably weaker and broader than the simulated peaks, as a result of damping and attenuation by the $\mathrm{Cr}$ adhesion layer, and inhomogeneous broadening caused by slight variations in the size and shape of each nanoparticle. The peak position of the $50 \mathrm{~nm}$ square is very close to the interband region, leading to a low extinction efficiency and a low radiative efficiency. The simulated spectra for the $50 \mathrm{~nm}$ and $100 \mathrm{~nm}$ squares have minor secondary peaks which are not present in the experimental spectra. These occur between approximately $550 \mathrm{~nm}$ and $600 \mathrm{~nm}$, where there also appears to be small fluctuations in the general trend of the values of $k$ in the optical constants data of $\mathrm{Au}$ tabulated by Palik. These fluctuations are not present in other sources of optical constants for $\mathrm{Au}$, and so are likely to be erroneous.

The experimental spectra of $\mathrm{Al}$ nanoparticles are in good agreement with both the simulated extinction peak position and efficiency. The peak position of the simulated $200 \mathrm{~nm}$ particle spectra is difficult to determine due to the flat-top, but was estimated as the midpoint of the plateau. The experimental spectrum of the $200 \mathrm{~nm}$ particle does not feature such a pronounced plateau. Unlike the noble metals, Al does not feature a distinct interband threshold at short wavelengths but instead features a narrow range of interband transitions centered at approximately $825 \mathrm{~nm}$. The interband region presents itself as a minor increase in the extinction spectra of the $100 \mathrm{~nm}$ and $150 \mathrm{~nm}$ squares at around $825 \mathrm{~nm}$. The extinction peak of the $200 \mathrm{~nm}$ squares occurs within the interband range, and so the center of the peak is attenuated, resulting in a flattened peak. (a)

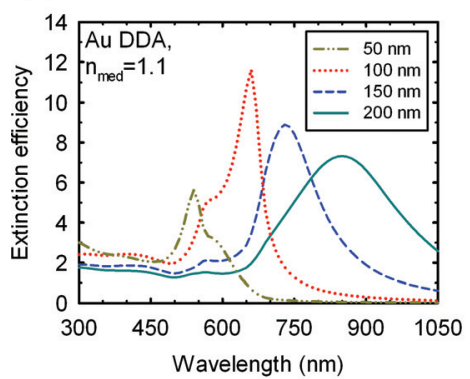

(c)

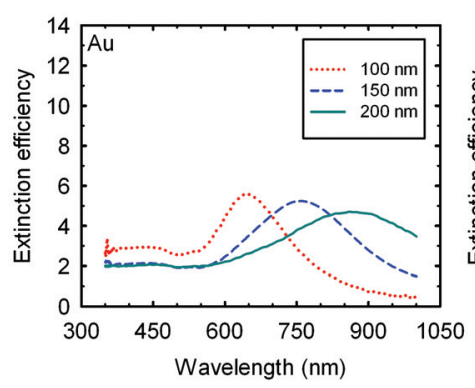

(b)

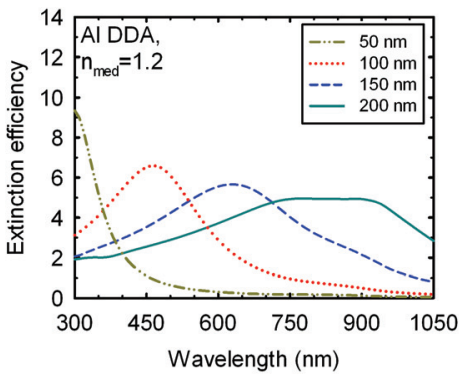

(d)

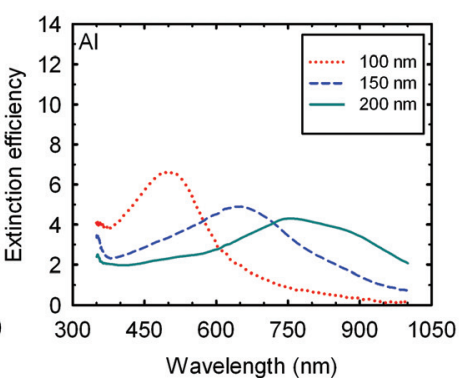

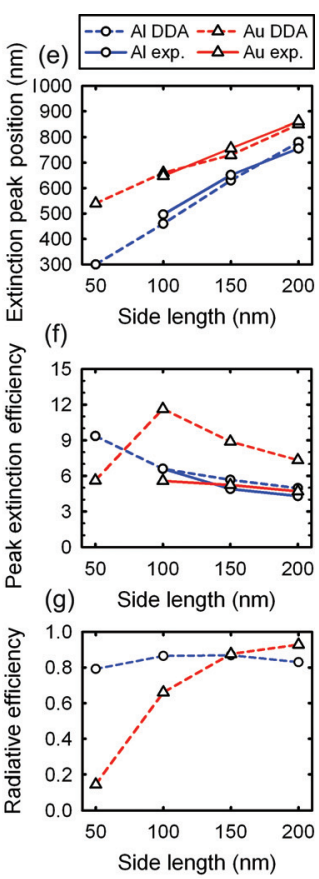

FIG. 7. (Color online) Optical properties of square metal nanoparticles with side lengths ranging from $50 \mathrm{~nm}$ to 200 nm. (a) Simulated extinction spectra of Au nanoparticles, (b) simulated extinction spectra of $\mathrm{Al}$ nanoparticles, (c) experimental extinction spectra of $\mathrm{Au}$ nanoparticles, (d) experimental extinction spectra of $\mathrm{Al}$ nanoparticles. (e) Summary of extinction peak positions, (f) summary of extinction efficiencies at the extinction peak, $(\mathrm{g})$ summary of radiative efficiency at the extinction peak. The out-of-plane height of the nanoparticles is $40 \mathrm{~nm}$ in all cases. 
With the exception of the smallest particle size, the simulated peak extinction efficiencies of Au nanoparticles are higher than $\mathrm{Al}$ nanoparticles of the same geometry. The simulated spectrum of the $100 \mathrm{~nm}$ Au particle demonstrates the strongest optical interaction of the square nanoparticles, with a peak extinction efficiency of 11.64. Meanwhile, the simulations indicate an extinction efficiency of 9.45 for the $50 \mathrm{~nm} \mathrm{Al}$ nanoparticles. This is the strongest interaction of the $\mathrm{Al}$ nanoparticles, but it occurs at a wavelength of 300 $\mathrm{nm}$, which is not useful for silicon solar cells. The reverse trend is found in the experimental spectra where the $\mathrm{Al}$ nanoparticles have a stronger interaction than the Au nanoparticles, because of the attenuation caused by the $\mathrm{Cr}$ adhesion layer. The effect of increasing the nanoparticle size on the peak position is stronger for $\mathrm{Al}$ nanoparticles than for $\mathrm{Au}$. For example, increasing the side length of a square nanoparticle from $50 \mathrm{~nm}$ to $200 \mathrm{~nm}$ results in a red-shift of the peak by $310 \mathrm{~nm}$ for Au and $480 \mathrm{~nm}$ for Al.

The simulation results show that radiative efficiencies increase with nanoparticle size, as is the case for spheres. ${ }^{26}$ Radiative efficiency is a function of size and also the choice of metal. The radiative efficiency of $\mathrm{Al}$ nanoparticles increases from 0.79 for a $50 \mathrm{~nm}$ particle to 0.87 for a $150 \mathrm{~nm}$ particle due to an increase in size, but then decreases to 0.83 for the $200 \mathrm{~nm}$ particle because the peak is shifted within the interband region of $\mathrm{Al}$. The change in radiative efficiency is much larger for $\mathrm{Au}$, with a minimum value of 0.15 for the 50 $\mathrm{nm}$ particle and a maximum value of 0.93 for the $200 \mathrm{~nm}$ particle. For small particles with resonances in the UV and visible $\mathrm{Al}$ has higher radiative efficiencies, while for larger particles with resonances in the NIR Au has a higher radiative efficiency. The crossover point occurs for the $150 \mathrm{~nm}$ particle, where a similar radiative efficiency is observed for both metals.

\section{Cross-sectional shape}

In addition to nanoparticle size, the cross-sectional shape and the three-dimensional shape also strongly affect the optical properties of metal nanoparticles. Haes et al. used FDTD simulations to show that, for a constant nanoparticle volume, the peak was red-shifted with increasing surface curvature, i.e. "sharp" tips red-shift the resonance position. ${ }^{38}$ For example, the extinction peak of a pyramid is considerably red-shifted in comparison with that of a sphere of the same volume. The shape of the nanoparticle affects the oscillation of the electrons and the near-field distribution of energy. Hence it is also important to investigate what effect particle shape has on radiative efficiency.

A number of different nanoparticle shapes can be made using low cost lithographic methods. For example, diskshaped nanoparticles can be fabricated using hole-mask colloidal lithography (HCL), ${ }^{39}$ and triangular (truncated tetrahedral) nanoparticles can be fabricated using nanosphere lithography (NSL). ${ }^{40}$

EBL allows a free choice of lateral particle shape, and so the influence of particle shape can be studied while keeping all other parameters such as size and surface coverage constant. In this section we compare the optical properties of

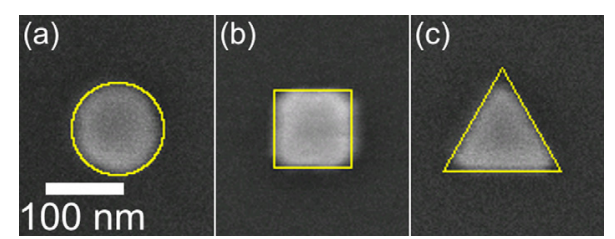

FIG. 8. (Color online) SEM images of typical nanoparticles fabricated by EBL to investigate shape: (a) circle with diameter $115 \mathrm{~nm}$, (b) square with side-length $100 \mathrm{~nm}$, (c) triangle with side-length $150 \mathrm{~nm}$. The yellow outlines show the designed dimensions, drawn to the same scale as the SEM image. All three shapes have the same cross-sectional area.

nanoparticles with three different cross-sectional shapes: circular, square and triangular. The dimensions were chosen such that all three types of nanoparticle have a cross-sectional area close to $10,000 \mathrm{~nm}^{2}$. The height was fixed at 40 $\mathrm{nm}$, and so all nanoparticles in this study have a similar volume. SEM images of exemplary particles of each type are shown in Fig. 8. The dimensions of the fabricated nanoparticles are close to those specified in the design, except for the rounding of the corners for the square and triangular nanoparticles. The optical properties of the arrays are presented in Fig. 9.

The cross-sectional shape is seen to affect the peak position, extinction efficiency, and radiative efficiency. The peak position is shifted to longer wavelengths as the cross-sectional shape is changed from circle to square to triangle, i.e., as the "edge sharpness" is increased. The overall change in peak position is relatively small, with a maximum shift of less than $80 \mathrm{~nm}$ for the simulated spectra, and less than 50 $\mathrm{nm}$ for the experimental spectra. The smaller shift for the experimental spectra is due to the rounding of the corners that occurs during fabrication. The square nanoparticle exhibits the strongest peak extinction efficiency for both metals, but the reason for this is not clear.

Additional spectral features at short wavelengths are seen for the simulated spectra for Au nanoparticles, which we again ascribe to possible errors in the optical constants data. The peak positions of the $\mathrm{Al}$ nanoparticles are far away from the interband region, but a slight increase in extinction around 750-950 $\mathrm{nm}$ can be seen in the simulated spectra of the triangular nanoparticle. Separating the simulated spectra into absorption and scattering plots (not shown) reveals that this slight increase in extinction is due to an increase of absorption in the $\mathrm{Al}$ interband region.

The radiative efficiencies of the $\mathrm{Al}$ square and circular particles are higher than the corresponding Au nanoparticles. This is because the extinction peaks of these shapes occur at short wavelengths, close to the Au interband region. For both metals the radiative efficiency is seen to increase as the cross-sectional shape is changed from circular to square, and this effect is strongest for $\mathrm{Al}$ with an increase from 0.74 to 0.86 . The triangular nanoparticles feature a considerably lower radiative efficiency than the other two geometries, with the Al triangular particle having a radiative efficiency of less than half that of a square particle of the same volume. The strong decrease is most likely due to the high field concentration generated at the tips of the particle, ${ }^{3,38}$ resulting in increased absorption. 
(a)

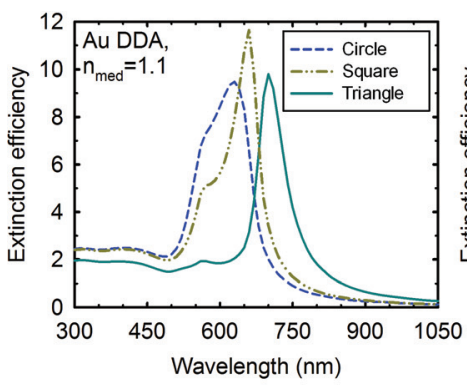

(c)

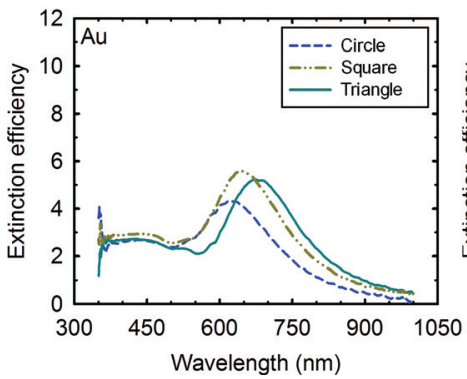

(b)

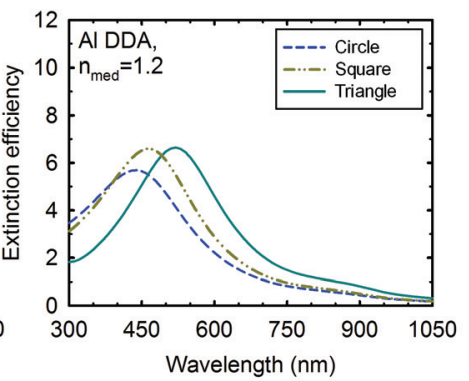

(d)

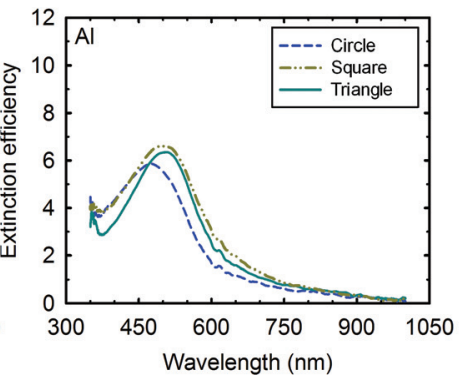

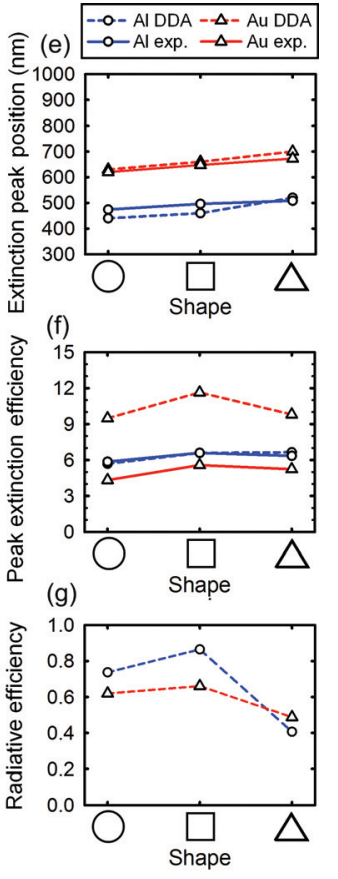

FIG. 9. (Color online) Optical properties of metal nanoparticles with circular, square and triangular cross-section. (a) Simulated extinction spectra of Au nanoparticles, (b) simulated extinction spectra of $\mathrm{Al}$ nanoparticles, (c) experimental extinction spectra of Au nanoparticles, (d) experimental extinction spectra of Al nanoparticles. (e) Summary of extinction peak positions, (f) summary of extinction efficiency at the extinction peak, (g) summary of radiative efficiency at the extinction peak. The out-of-plane height of the nanoparticles is $40 \mathrm{~nm}$ in all cases. The cross-sectional area, and hence the volume, is the same for all three nanoparticles.
The tips of the fabricated nanoparticles are truncated due to limitations in pattern definition during exposure, and rounding of the features during resist development and metal deposition. Kelly et al. investigated the effect of tip truncation on triangular Ag nanoparticles using DDA simulations, and found that it resulted in a strong blue-shifting of the extinction peak. ${ }^{3}$ In Fig. 10 we present results from DDA simulations that show the effect of tip truncation on the peak position and the radiative efficiency. The effect of truncating the tip is to blue-shift the extinction peak position and increase the radiative efficiency. The blueshift is a result of both the decreased particle size and the tip truncation. However, a decrease in particle size is expected to decrease the radiative efficiency, ${ }^{32}$ and so the increase in radiative efficiency is due only to the tip truncation, most likely due to a reduction of field concentration at the tips. The truncation

(a)

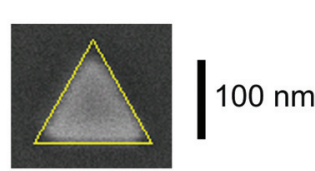

(c)
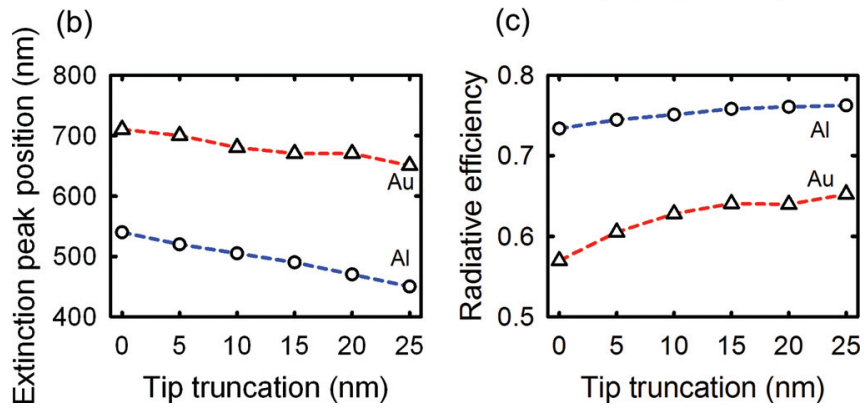

FIG. 10. (Color online) (a) SEM image of a triangular nanoparticle with truncated tips, summary of simulated (b) extinction peak positions and (c) radiative efficiency for truncated $\mathrm{Au}$ and $\mathrm{Al}$ triangles with an initial side length of $150 \mathrm{~nm}$. performed in the simulations is a crude simplification of the rounding that occurs during fabrication, but this study demonstrates that the optical properties of triangular nanoparticles are highly sensitive to the tip geometry.

\section{E. Aspect ratio}

Anisotropic nanoparticles support different LSPs depending on the polarization state of the incident light. The simplest anisotropic geometry is a prism with a rectangular cross-section, which we will refer to as a nanorod. Nanorods support two orthogonal LSPs: a longitudinal (long-axis) mode and a transverse (short-axis) mode. Each mode can be excited independently by varying the polarization of incident light. Both modes are excited equally when exposed to unpolarized light.

The aspect ratio, defined as the ratio of the longest side to the shortest side, has a strong influence on the optical properties of nanorods, with an increase in aspect ratio resulting in a red-shift of the extinction peak. ${ }^{33,41}$ Numerous studies have investigated the influence of aspect ratio on the extinction magnitude and peak position, using both simulations and experiments, but the influence of nanorod dimensions on radiative efficiency has received less attention, ${ }^{41}$ and to our knowledge $\mathrm{Al}$ nanorods have not yet been studied experimentally.

Nanorods with very high aspect ratios have been shown to support many higher-order modes, ${ }^{42-44}$ with the fundamental dipolar mode occurring far beyond the range required for silicon photovoltaics. Instead, we investigate modest aspect ratios ranging from 1 to 4 , with the aim of achieving dipolar extinction peaks in the $400-1000 \mathrm{~nm}$ range. In this experiment the rectangle width was kept constant at $50 \mathrm{~nm}$, while the length was varied from $50 \mathrm{~nm}$ to $200 \mathrm{~nm}$. It should be noted that, in contrast to the previous section, the crosssectional area of the nanorods was not kept constant. 

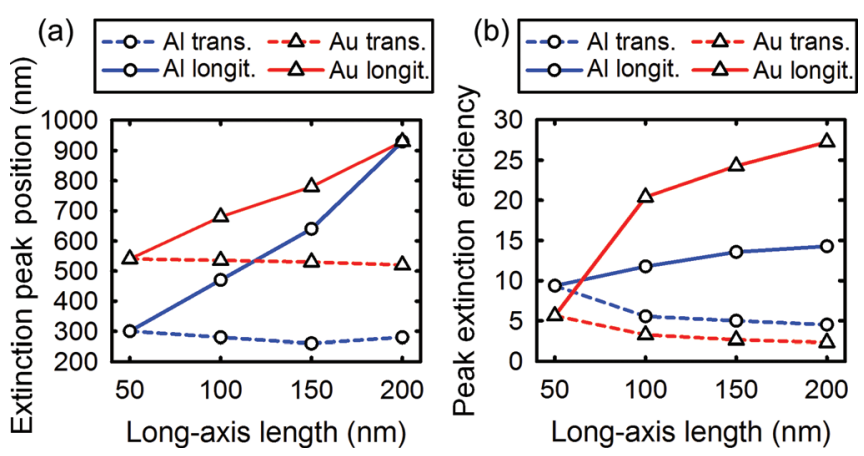

FIG. 11. (Color online) Simulated (a) peak postions and (b) extinction efficiency at the extinction peak, for $\mathrm{Au}$ and $\mathrm{Al}$ nanorods with minor side length $50 \mathrm{~nm}$ and major side length range from $50 \mathrm{~nm}$ to $200 \mathrm{~nm}$, corresponding to aspect ratios ranging from 1 to 4 . The transverse mode is excited by photons polarized parallel to the short axis, and the longitudinal mode is excited by photons polarized parallel to the long axis.

Therefore, the changes in optical properties are related to both aspect ratio and cross-sectional size.

For solar cell applications we need only consider the response of metal nanoparticles to unpolarized light, but we will briefly discuss the two nanorod modes separately to highlight their unique optical properties in comparison with the geometries discussed in the previous sections. The influence of rod length on the longitudinal and transverse modes can be seen in the simulation results presented in Fig. 11. For both metals the longitudinal mode is strongly red-shifted by increasing the length of the nanorod, while the transverse mode is slightly blue-shifted. The transverse mode is considerably weaker than the longitudinal mode, and the difference in extinction efficiencies between the two modes increases with rod length. The overall polarization sensitivity is also remarkably high, particularly at the longitudinal extinction peak position of the longest nanorod. For example, at a wavelength of $930 \mathrm{~nm}$, a $50 \mathrm{~nm}$ by $200 \mathrm{~nm}$ Au nanoparticle exhibits an extinction efficiency of 27.26 for light polarized parallel to the long axis (longitudinal mode), and an extinction efficiency of 0.04 for light polarized parallel to the short axis (transverse mode).

Polarization sensitivity is not desirable for solar cell applications because we wish to maximize interaction with all incident photons. An array of nanorods will only be sensitive to polarization if all of the nanorods are aligned in the same direction. Randomizing the orientation of nanorods within an array will result in an array that has the same optical properties for all incident polarizations (assuming the illumination angle is perpendicular to the substrate plane). The simulation results given in Fig. 11 were for light polarized parallel to one of the particle axes. For unpolarized light incident on an array of randomly orientated nanorods the net response will be equal to the average of both polarization states. For longer nanorods this has the effect of nearly halving the logitudinal peak extinction efficiency, although these average values can still be very large.

The fabricated rectangular nanoparticles are shown in Fig. 12. The length and the width of the nanoparticles are close to the designed values, but some edge rounding is evident. The $50 \mathrm{~nm}$ particles were not fabricated successfully

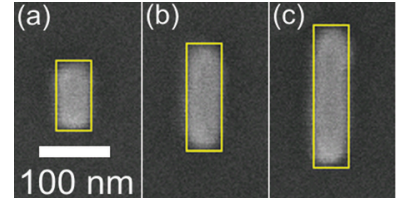

FIG. 12. (Color online) SEM images of typical nanoparticles fabricated by EBL to investigate aspect ratio. All four shapes have an out-of-plane height of $40 \mathrm{~nm}$ and a width of $50 \mathrm{~nm}$ The lengths are (a) $100 \mathrm{~nm}$, (b) $150 \mathrm{~nm}$, and (c) $200 \mathrm{~nm}$. These values correspond to aspect ratios ranging from 2 to 4 . The yellow outlines show the designed dimensions, drawn to the same scale as the SEM image.

and so are only included in the simulation results. The experimental and simulated optical spectra of rectangular nanoparticles, for the case of unpolarized light, are shown in Fig. 13.

The extinction spectra of all nanorods are clearly dominated by the extinction peak arising from the longitudinal LSP mode. The peak value of this mode is halved in comparison with the results given in Fig. 11, due to averaging over two polarizations. Even so, the peak extinction values are in some cases higher than those of square nanoparticles with similar peak positions, and the simulated spectrum of the $200 \mathrm{~nm}$ long Au nanorod features the highest extinction efficiency of any of the geometries presented in this study. The transverse mode cannot be observed in any of the $\mathrm{Al}$ spectra because it occurs at wavelengths below $300 \mathrm{~nm}$. The transverse modes can be seen in the Au spectra as small peaks around $520 \mathrm{~nm}$ to $540 \mathrm{~nm}$, most noticeably for the $100 \mathrm{~nm}$ long particle. For photovoltaic applications the transverse modes can be considered as negligible in comparison with the longitudinal modes, due to their low extinction efficiency and short wavelength peak positions. As such, we will only discuss the trends of the longitudinal mode in the remainder of this section.

An increase in aspect ratio is seen to lead to a nearly linear red-shifting of the primary peak positions for both materials. The simulated peak positions are in good agreement with the experimental results, with the exception of the highest aspect ratio aluminum nanoparticle, due to a similar "plateau" peak shape to that observed for the $200 \mathrm{~nm}$ square nanoparticle. The simulated extinction spectrum of the longest rectangle appears to have two peaks, but analysis of the separate absorption and scattering spectra (not shown) show that this peak structure is due to suppression of scattering at wavelengths near the interband region. Interband transitions increase absorption and decrease scattering, and so appear as a depression in a peak within this area, or a local increase for peaks that are outside of the region.

The effect of tuning the extinction peak position by changing the nanorod length is similar to that achieved by increasing the size of square nanoparticles: both methods result in extinction peaks across a similar range of wavelengths. However, increasing the aspect ratio also increases the peak extinction efficiency, while increasing the size of square particles decreases the peak extinction efficiency. The radiative efficiency trends for nanorods are similar to those observed for squares in Fig. 7, with $\mathrm{Al}$ having the highest radiative efficiency at short wavelengths, and Au having the 
(a)

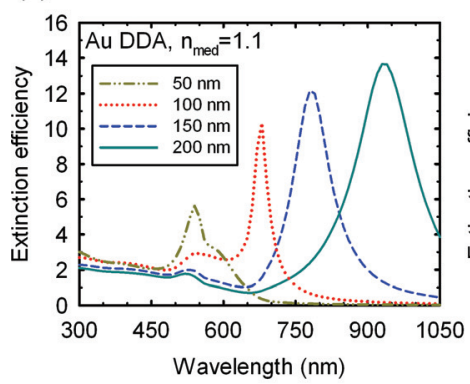

(c)

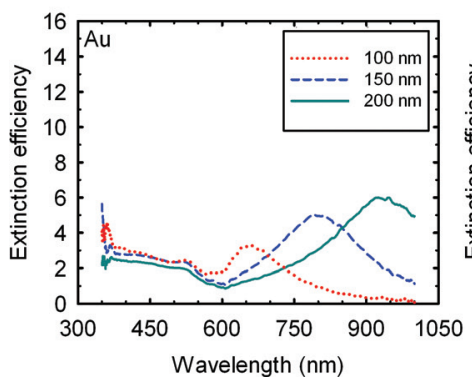

(b)

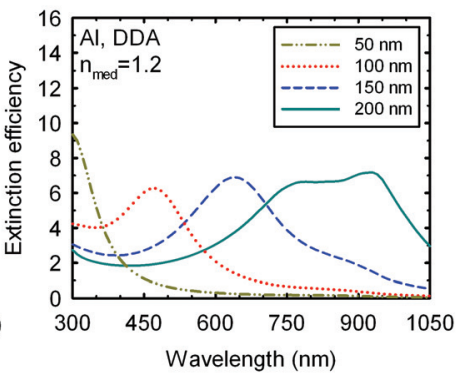

(d)

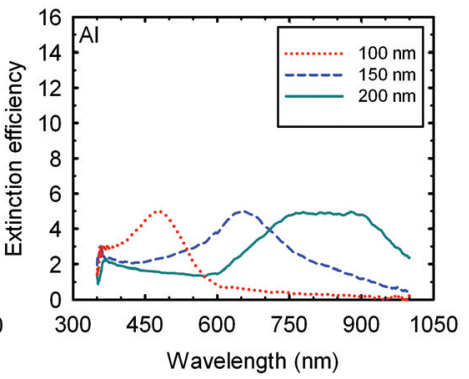

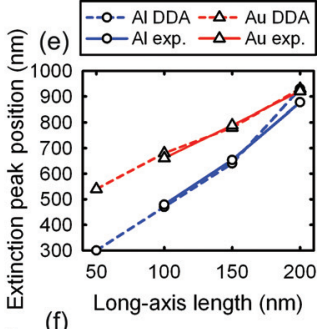

(f)

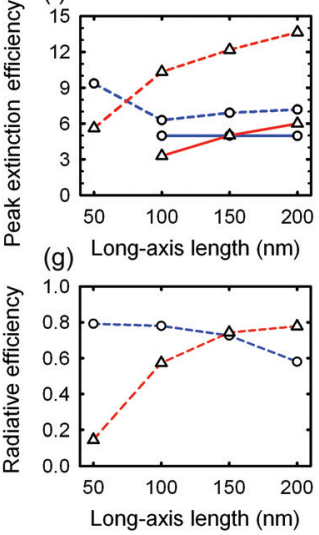

FIG. 13. (Color online) Optical properties of rectangular metal nanoparticles with minor side length of $50 \mathrm{~nm}$, and major side lengths ranging from $50 \mathrm{~nm}$ to $200 \mathrm{~nm}$. (a) Simulated extinction spectra of $\mathrm{Au}$ nanoparticles, (b) simulated extinction spectra of $\mathrm{Al}$ nanoparticles, (c) experimental extinction spectra of Au nanoparticles, (d) experimental extinction spectra of Al nanoparticles. (e) Summary of longitudinal extinction peak positions, (f) summary of extinction efficiencies at the longitudinal extinction peak, (g) summary of radiative efficiency at the longitudinal extinction peak. The out-of-plane height of the nanoparticles is $40 \mathrm{~nm}$ in all cases. The simulations are the average of two orthogonal polarizations. highest radiative efficiencies at long wavelengths. However, the square particles have a higher radiative efficiency overall.

\section{F. Metal}

So far we have focused on the optical properties of nanoparticles made from $\mathrm{Au}$ or $\mathrm{Al}$, but $\mathrm{Ag}$ and $\mathrm{Cu}$ can also be used for plasmonic applications in the visible and NIR. In this section we will compare trends of the simulated optical properties of the same nanorods discussed in Sec. IV E, but extended to include $\mathrm{Ag}$ and $\mathrm{Cu}$. The optical constants of $\mathrm{Ag}$ and $\mathrm{Cu}$ were obtained from Palik. ${ }^{17} \mathrm{Ag}$ and $\mathrm{Cu}$ nanoparticles were not fabricated and so it was not possible to determine the effective medium values to account for oxide layers on these metals. Instead, all four metals were simulated in an effective medium with a refractive index of 1.2. A summary of the trends for Al, $\mathrm{Ag}, \mathrm{Au}$, and $\mathrm{Cu}$ nanorods is shown in Fig. 14.

Noble metal-Ag, $\mathrm{Au}$ and $\mathrm{Cu}$-nanorods exhibit very similar trends in extinction peak position, peak efficiency, and radiative efficiency. The optical constants of these three metals are similar for wavelengths of approximately $700 \mathrm{~nm}$ and above. The differences at shorter wavelengths are due to variation in the energies at which interband transitions become important. The interband threshold of Ag occurs at a shorter wavelength than $\mathrm{Au}$ and $\mathrm{Cu}$, and so $\mathrm{Ag}$ nanoparticles can support resonances at shorter wavelengths than $\mathrm{Au}$ and $\mathrm{Cu}$. In general the extinction efficiency and radiative efficiency decrease in the order of $\mathrm{Ag}$, $\mathrm{Au}$, and then $\mathrm{Cu}$. Smaller $\mathrm{Cu}$ nanoparticles behave similarly to $\mathrm{Au}$, but at longer wavelengths the difference in radiative efficiency becomes larger. We note that this could be due to an erroneous point in the optical constants data, as very few data points are given by Palik for $\mathrm{Cu}$ in this wavelength range.

The trends for $\mathrm{Al}$ are markedly different to those of the noble metals. The similarities between the noble metals means that the comparisons made between $\mathrm{Al}$ and $\mathrm{Au}$ in the previous sections can also be extended to $\mathrm{Ag}$ and $\mathrm{Cu}$, except for the fact that the cross-over point at which $\mathrm{Al}$ has the lowest radiative efficiency occurs at a shorter rod length for $\mathrm{Ag}$, and a longer rod length for $\mathrm{Cu}$.

For $\mathrm{Al}$ and $\mathrm{Ag}$ nanorods the extinction efficiency is seen to decrease as the long-axis length is increased from $50 \mathrm{~nm}$ to $100 \mathrm{~nm}$, because of the splitting of the excitation between the two orthogonal modes. For $\mathrm{Au}$ and $\mathrm{Cu}$ an increase in extinction efficiency is seen for the same conditions, because increasing the long-axis length shifts the resonance away from the interband region.

In addition to optical properties, it is important to also consider the material properties of the four metals. The noble metals have poor adhesion to most substrates, but this is only important for fabrication methods that require a chemical lift-off process. The rapid diffusion of $\mathrm{Au}$ atoms into semiconductors can result in deep-level energy states that negatively affect device performance. The risk of $\mathrm{Au}$ diffusion can be reduced by ensuring that nanoparticles are only deposited after any high temperature process steps, and also by the use of barrier layers ( such as $\mathrm{SiO}_{2}$ ).

$\mathrm{Al}, \mathrm{Ag}$ and $\mathrm{Cu}$ all form a surface oxide layer on exposure to air, each with different optical properties. $\mathrm{Al}_{2} \mathrm{O}_{3}$ is non-absorbing in the visible and NIR and does not negatively affect the excitation of LSPs, while $\mathrm{AgO}_{\mathrm{x}}$ has nonzero absorption in this range, which may lead to damping of the LSP excitation in a similar manner to the $\mathrm{Cr}$ adhesion layers used in this study. $\mathrm{Cu}_{2} \mathrm{O}$ has been shown to shift the peak position of $\mathrm{Cu}$ nanoparticles, but does not appear to have any negative effects. ${ }^{45}$ Further work is required to study the formation and influence of oxide layers on $\mathrm{Al}, \mathrm{Ag}$, and $\mathrm{Cu}$ nanoparticles.

Finally, the material cost and abundance are clearly important when considering the use of metal nanoparticle in 


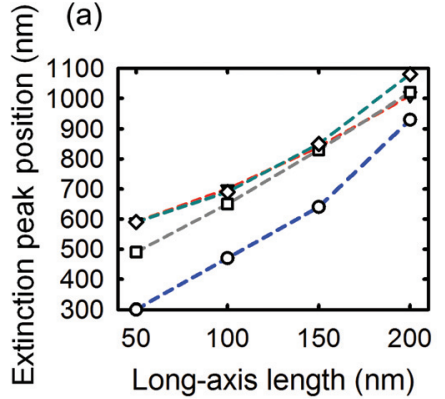

(b)

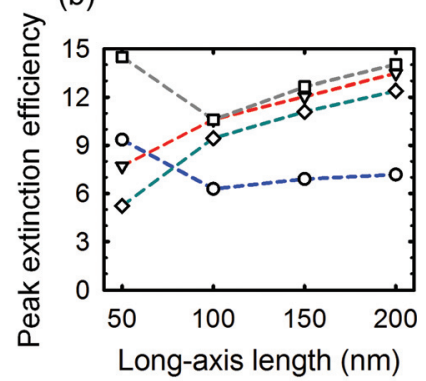

(c)

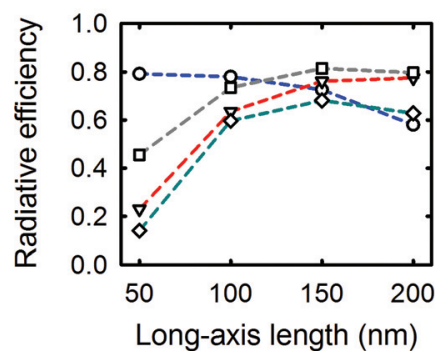

FIG. 14. (Color online) (a) Simulated peak postions, (b) extinction efficiency at the extinction peak, and (c) radiative efficiency at the extinction peak, of nanorods with minor side length $50 \mathrm{~nm}$ and major side length ranging from $50 \mathrm{~nm}$ to $200 \mathrm{~nm}$, for $\mathrm{Al}, \mathrm{Ag}$, $\mathrm{Au}$, and $\mathrm{Cu}$, embedded in a homogenous dielectric medium with a refractive index of 1.2.

solar cells, with $\mathrm{Al}$ being by far the cheapest and most abundant of the four metals. However, given that substantially less than a monolayer of nanoparticles are required, it seems likely that the cost and efficiency of the nanoparticle fabrication method will be more important than the cost of the metal itself.

\section{G. Summary and Discussion}

The results presented above demonstrate that the optical properties of metal nanoparticles are strongly dependent on the size, shape, and the type of metal used. For light-trapping in silicon solar cells, we require nanoparticles with a high extinction efficiency, a high radiative efficiency, and an extinction peak close to where the semiconductor is most weakly absorbing. All three parameters are affected by changes to the particle size, shape or metal, and so care must be taken so that achieving one goal does not come at the cost of another.

The extinction peak position of a metal nanoparticle can be red-shifted by increasing lateral size, aspect-ratio or tip curvature. We have demonstrated extinction peak positions up to $930 \mathrm{~nm}$ for both $\mathrm{Al}$ and $\mathrm{Au}$ nanoparticles. Extinction efficiencies beyond this are possible by further increasing the size, aspect-ratio or the refractive index of the surrounding medium. Tuning the peak position by increasing the size results in broad but relatively weak extinction efficiencies with high radiative efficiencies. Tuning the peak position by aspect ratio results in much higher extinction efficiencies, but lower radiative efficiencies. Nanoparticles with high degrees of curvature (i.e., sharp tips) should be avoided for scattering-based enhancement of inorganic solar cells, because of their low radiative efficiency.
The choice of metal has a strong influence on the optical properties of particles. The results presented in the previous sections demonstrate that $\mathrm{Au}$ and $\mathrm{Al}$ nanoparticles with identical geometries can behave in very different ways. Nanoparticles made from either metal are capable of supporting LSPs in the visible and NIR, with the range extended into the UV for Al nanoparticles. The extinction peaks of Al nanoparticles are broader and weaker than corresponding Au nanoparticles, with the exception of Au nanoparticles that have extinction peaks near the Au interband threshold. Al exhibits a higher radiative efficiency in the UV and visible, while Au exhibits a higher radiative efficiency in the NIR. The poor radiative efficiency in both cases is due to interband transitions, which occur at different energies for each metal. The Au interband region has a stronger negative effect on extinction efficiency and radiative efficiency than the $\mathrm{Al}$ interband region. $\mathrm{Ag}$ and $\mathrm{Cu}$ nanoparticles exhibit similar trends to $\mathrm{Au}$, with $\mathrm{Ag}$ nanoparticles capable of supporting resonances at shorter wavelengths, and having a higher radiative efficiency. The role of surface oxide formation is an important consideration that requires further study.

For amorphous silicon solar cells we require strong scattering in the 500-750 $\mathrm{nm}$ range, and so aluminum nanoparticles will be most suitable due to their higher radiative efficiency in this range. The interband region of $\mathrm{Al}$ occurs below the bandgap of a-Si:H and so need not be considered. Of the geometries studied the $150 \mathrm{~nm}$ square $\mathrm{Al}$ nanoparticle is the most suitable for amorphous silicon applications, with an extinction peak position of $630 \mathrm{~nm}$ and a radiative efficiency of 0.87 .

For multicrystalline or crystalline silicon devices $\mathrm{Au}$ and $\mathrm{Ag}$ are the most suitable, as they have the highest radiative efficiency in the $900-1100 \mathrm{~nm}$ range. The $200 \mathrm{~nm}$ square Au nanoparticle has a peak position of $850 \mathrm{~nm}$ and a radiative efficiency of 0.93 , and achieves this without introducing significant surface roughness. However, metallic adhesion layers cannot be used for Au nanoparticles due to the considerable decrease in radiative efficiency that they introduce. Alternatives include the use of an organic adhesion layers, ${ }^{46,47}$ or a milder lift-off process that does not requrie an adhesion layer.

The magnitude of the extinction efficiency is less important than the peak position and the radiative efficiency for solar cell applications but must still be taken into consideration. The extinction efficiency is the ratio of the optical cross-section to the geometric cross-section, and so it determines the minimum surface coverage of nanoparticles that is required to interact with (i.e., scatter or absorb) all incident photons. High extinction efficiencies mean that fewer particles are required, and so the materials costs are reduced. Additionally, high extinction efficiencies also introduce the possibility of combining multiple particle types into a single array, to broaden the range of wavelengths that are scattered. ${ }^{48}$ For example two nanoparticle types with extinction efficiencies of 10 can be combined into a single array with a total surface coverage of only $20 \%$. Nanorods enable tuning of the peak position while maintaining extremely high extinction efficiencies, and so are the most suitable nanoparticle type for multiple particle ensembles. For arrays 
with a high surface coverage the effect of interparticle coupling must be taken into consideration, and single particle simulations will no longer provide an accurate prediction of the optical properties of the entire array.

Correctly designed metal nanoparticles can strongly scatter NIR photons while maintaining the low surface roughness that is required for high quality semiconductor film growth. The lithographically defined nanoparticles presented in this article lead to a much lower surface roughness than typical metal nanoparticle fabrication methods such as metal island films or spherical particles prepared by chemical synthesis. Similar geometries to those presented in this paper can be achieved by low cost fabrication methods such as colloidal lithography ${ }^{39,40}$ or chemical synthesis. ${ }^{49,50}$ The maximum peak-to-peak height is the height of the nanoparticles, $40 \mathrm{~nm}$ in this case, and the rms roughness depends on the surface coverage. Spherical particles require large diameters to obtain extinction peaks in the NIR and also have considerably weaker extinction efficiencies. ${ }^{26}$

The results presented here represent only a small part of the total parameter space available for metal nanoparticles. Additional parameters should be investigated to fully optimize the design of metal nanoparticles for photovoltaic applications. These parameters include the out-of-plane height ${ }^{51}$ and the local dielectric environment (e.g., the layers normally present in a solar cell, which would surround the particles). We note that the geometry studied in this workmetal nanoparticles on fused-silica substrates-represents the minimum value of surrounding medium refractive index that can be expected. This value will be considerably higher when the nanoparticles are integrated into a solar cell due to proximity to a semiconductor layer, and encapsulation within a dielectric layer (for example $\mathrm{SiO}_{2}$, EVA, or a transparent conductive oxide). The effect of increasing the surrounding medium will be to further red-shift the resonance position, and so the results presented in this study should be considered as the minimum peak position that is achievable for a given nanoparticle geometry.

\section{CONCLUSION}

Metal nanoparticles are extremely versatile optical engineering tools because of their intense and tuneable interaction with light. However, this interaction must be correctly tailored to suit a given application. We have demonstrated a good match between experimental results for large particle arrays with DDA simulations of isolated nanoparticles. This was in part achieved by approximating the complex dielectric environment surrounding the nanoparticles as a simple homogenous medium with a refractive index of 1.2 for $\mathrm{Al}$ nanoparticles and 1.1 for Au nanoparticles. Simulations can be used to explore a wider parameter space to fully optimize the optical properties of metal nanoparticles for photovoltaic applications.

We have demonstrated that the extinction peaks of $\mathrm{Au}$ and $\mathrm{Al}$ metal nanoparticles can be tuned across the solar spectrum by modifying the lateral size and shape. Al nanoparticles have broader and weaker peaks than corresponding $\mathrm{Au}$ nanoparticles, but still support well-defined resonances across the entire solar spectrum. Planar metal nanoparticles offer several advantages over spherical particles for PV applications, including an increased extinction efficiency and a lower overall substrate roughness. The peak position of planar metal nanoparticles can be tuned across the entire solar spectrum without increasing the nanoparticle height, and hence without increasing the peak to peak roughness of the substrate.

For photovoltaic applications it is particularly important to minimize absorption (i.e., maximize the radiative efficiency), and this can be achieved by avoiding small particles and geometries with high degrees of curvature. Additionally, metallic adhesion layers such as $\mathrm{Cr}$ should not be used.

To date there has been little effort to optimize the size, shape and material of metal nanoparticles for photovoltaic applications. However, incorrectly designed nanoparticles can result in sub-optimum enhancement, or even reduction of device efficiency. The challenge remains to establish the optimum nanoparticle parameters for each solar cell type, and to find a suitable low-cost, large area fabrication technique.

\section{ACKNOWLEDGMENTS}

The authors wish to acknowledge NIL Technology, Copenhagen for fabrication of the metal nanoparticle arrays.

${ }^{1}$ E. Hutter and J. H. Fendler, Adv. Mater. 16, 1685 (2004).

${ }^{2}$ W. A. Murray and W. L. Barnes, Adv. Mater. 19, 3771 (2007).

${ }^{3}$ K. L. Kelly, E. A. Coronado, L. L. Zhao, and G. C. Schatz, J. Phys. Chem. B 107, 668 (2003).

${ }^{4}$ K. A. Willets and R. P. Van Duyne, Ann. Rev. Phys. Chem. 58, 267 (2007).

${ }^{5}$ J. N. Anker, W. P. Hall, O. Lyandres, N. C. Shah, J. Zhao, and R. P. Van Duyne, Nature Mater. 7, 442 (2008).

${ }^{6}$ C. Hägglund and B. Kasemo, Opt. Express 17, 11944 (2009).

${ }^{7}$ H. A. Atwater and A. Polman, Nat. Mater. 9, 205 (2010).

${ }^{8}$ S. H. Lim, W. Mar, P. Matheu, D. Derkacs, and E. T. Yu, J. Appl. Phys. 101, 104309 (2007).

${ }^{9}$ H. R. Stuart and D. G. Hall, Appl. Phys. Lett. 69, 2327 (1996).

${ }^{10}$ S. Pillai, K. R. Catchpole, T. Trupke, and M. A. Green, J. Appl. Phys. 101, 093105 (2007).

${ }^{11}$ E. Moulin, J. Sukmanowski, P. Luo, R. Carius, F. X. Royer, and H. Stiebig, J. Non-Cryst. Solids, 354, 2488 (2008).

${ }^{12}$ C. Hägglund, M. Zäch, G. Petersson, and B. Kasemo, Appl. Phys. Lett., 92, 053110 (2008).

${ }^{13}$ T. L. Temple, G. Mahanama, H. Reehal, and D. M. Bagnall, Sol. Energy Mater. Sol. Cells 93, 1978 (2009).

${ }^{14}$ Y. Nasuno, M. Kondo, and A. Matsuda, Jpn. J. Appl. Phys. 40, L303 (2001).

${ }^{15}$ M. Python, E. Vallat-Sauvain, J. Bailat, D. Domine, L. Fesquet, A. Shah, and C. Ballif, J. Non-Cryst. Solids 354, 2258 (2008).

${ }^{16}$ P. B. Johnson and R. W. Christy, Phys. Rev. B 6, 4370 (1972).

${ }^{17}$ E. D. Palik, Handbook of Optical Constants of Solids, (Academic Press, New York, 1985).

${ }^{18}$ M. G. Blaber, M. D. Arnold, and M. J. Ford, J. Phys. Chem. C 113, 3041 (2009).

${ }^{19}$ C. Langhammer, B. Kasemo, I. Zoriác, J. Chem. Phys. 126, 194702 (2007).

${ }^{20}$ G. H. Chan, J. Zhao, G. C. Schatz, and R. P. Van Duyne, J. Phys. Chem. C 112, 13958 (2008).

${ }^{21}$ T. Dörfer, M. Schmitt, and J. Popp, J. Raman Spectrosc. 38, 1379 (2007).

${ }^{22}$ A. Taguchi, N. Hayazawa, K. Furusawa, H. Ishitobi, S. Kawata, J. Raman Spectrosc. 40, 1097 (2009).

${ }^{23}$ M. H. Chowdhury, K. Ray, S. K. Gray, J. Pond, and J. R. Lakowicz, J. Anal. Chem. 81, 1397 (2009).

${ }^{24}$ Y. A. Akimov and W. S. Koh, Nanotechnol. 21, 235201 (2010).

${ }^{25}$ C. Langhammer, M. Schwind, B. Kasemo, and I. Zorić, Nano Lett. 8, 1461 (2008). 
${ }^{26}$ D. D. Evanoff and G. Chumanov, J. Phys. Chem. B 108, 13957 (2004).

${ }^{27}$ T. Jensen, K. L. Kelly, A. Lazarides, and G. C. Schatz, J. Clus. Sci. 10, 295 (1999)

${ }^{28}$ Y. B. Zheng, B. K. Juluri, X. Mao, T. R. Walker, and T. J. Huang, J. Appl. Phys. 103, $014308(2008)$

${ }^{29}$ E. M. Purcell, C. R. Pennypacker, Astrophys. J. 186, 705 (1973).

${ }^{30}$ B. T. Draine, P. J. Flatau, J. Opt. Soc. Am. A 11, 1491 (1994).

${ }^{31}$ B. T. Draine and P. J. Flatau, User Guide to the Discrete Dipole Approximation Code DDSCAT 6.1, available at http://arxiv.org/abs/astro-ph/ 0409262v2 (2004).

${ }^{32}$ C. F. Bohren and D. R. Huffman, Absorption and Scattering of Light by Small Particles (Wiley, New York, 2004).

${ }^{33}$ E. Stefan Kooij and B. Poelsema, Phys. Chem. Chem. Phys. 8, 3349 (2006).

${ }^{34}$ B. Auguié and W. L. Barnes, Opt. Lett. 34, 401 (2009).

${ }^{35}$ F. J. Garca De Abajo, Rev. Mod. Phys. 79, 1267 (2007).

${ }^{36}$ S. Zou and G. C. Schatz, J. Chem. Phys. 121, 12606 (2004).

${ }^{37}$ B. Auguié and W. L. Barnes, Phys. Rev. Lett. 101, 143902 (2008).

${ }^{38}$ A. Haes, C. Haynes, A. McFarland, G. C. Schatz, R. P. Van Duyne, and S. Zou, MRS Bull. 30, 369 (2005).
${ }^{39}$ H. Fredriksson, Y. Alaverdyan, A. Dmitriev, C. Langhammer, D. S. Sutherland, M. Zäch, and B. Kasemo, Adv. Mater. 19, 4297 (2007).

${ }^{40}$ J. Hulteen and R. Van Duyne, J. Vac. Sci. Technol. A 13, 1553 (2007).

${ }^{41}$ K. -S. Lee and M. A. El-Sayed, J. Phys. Chem. B 109, 20331 (2005).

${ }^{42}$ J. R. Krenn, G. Schider, W. Rechberger, B. Lamprecht, A. Leitner, F. R. Aussenegg, and J. C. Weeber, Appl. Phys. Lett. 77, 3379 (2000).

${ }^{43}$ E. K. Payne, K. L. Shuford, S. Park, G. C. Schatz, and C. A. Mirkin, J. Phys. Chem. B 110, 2150 (2006).

${ }^{44}$ B. N. Khlebtsov, A. Melnikov, and N. G. Khlebtsov, J. Quant. Spectrosc. Radiat. Transfer 107, 306 (2007).

${ }^{45}$ G. H. Chan, J. Zhao, E. M. Hicks, G. C. Schatz, and R. P. Van Duyne, Nano Lett. 7, 1947 (2007)

${ }^{46}$ C. A. Goss, D. H. Charych, and M. Majda, J. Anal. Chem. 63, 85 (1991).

${ }^{47}$ A. K. Mahapatro, A. Scott, A. Manning, and D. B. Janes, Appl. Phys. Lett. 88, 151917 (2006).

${ }^{48}$ J. R. Cole and N. J. Halas, Appl. Phys. Lett. 89, 153120 (2006).

${ }^{49}$ B. Nikoobakht and M. A. El-Sayed, Chem. Mater. 15, 1957 (2003).

${ }^{50}$ M. -C. Daniel and D. Astruc, Chem. Rev. 104, 293 (2004).

${ }^{51}$ J. Henson, J. DiMaria, and R. Paiella, J. Appl. Phys. 106, 093111 (2009). 